\title{
Coagulation Factor XII Gene Mutation in Brazilian Families with Hereditary Angioedema with Normal C1 Inhibitor
}

\author{
Adriana S. Moreno ${ }^{a, c}$ Solange O.R. Valle ${ }^{d}$ Soloni Levy ${ }^{d}$ Alfeu T. França ${ }^{d}$ \\ Faradiba S. Serpa ${ }^{e}$ Helen A. Arcuri ${ }^{f}$ Mario S. Palma ${ }^{\text {g }}$ Wagner N. Campos ${ }^{a}$ \\ Marina M. Dias a Denise Ponard ${ }^{h}$ Nicole Monnier ${ }^{h}$ Joel Lunardi ${ }^{h}$ \\ Konrad Bork ${ }^{i}$ Wilson Araujo Silva, Jr. ${ }^{b, c}$ L. Karla Arrudaa, ${ }^{a}$
}

Departments of ${ }^{a}$ Medicine and ${ }^{b}$ Genetics, Ribeirão Preto Medical School and ${ }^{c}$ Center for Medical Genomics, Clinical Hospital of Ribeirão Preto Medical School, University of São Paulo, Ribeirão Preto, ${ }^{d}$ Clementino Fraga Filho University Hospital, Federal University of Rio de Janeiro, Rio de Janeiro, e School of Medicine of Santa Casa de Misericórdia of Vitória, Espírito Santo, ${ }^{\mathrm{f}}$ School of Medicine of the University of São Paulo, São Paulo, and 9 Institute of Biosciences of Rio Claro, State University of São Paulo, Rio Claro, Brazil; h Joseph-Fourier University of Grenoble, Grenoble, France; 'Johannes-Gutenberg University, Mainz, Germany

\section{Key Words}

Hereditary angioedema - Coagulation factor XII .

C1 inhibitor

\begin{abstract}
Background: Hereditary angioedema (HAE) with normal C1 inhibitor $(\mathrm{C} 1-\mathrm{INH})$ is a rare disorder. Mutations of the gene encoding coagulation factor XII have been identified in a subset of patients with this condition. Our aim was to investigate mutations in the $F 12$ gene in patients with HAE with normal C1-INH from Brazil. Methods: We studied 5 Brazilian families with index female patients who presented with recurrent angioedema with normal C1-INH and C4 levels. Genomic DNA was isolated from whole blood and PCR was performed. Mutations were detected by the sequencing of exon 9 of the $F 12$ gene and allelic discrimination. Results: The c.983C >A (p.Thr328Lys) mutation was identified in 16 subjects, from 4 of the 5 families studied, including 8 pa-
\end{abstract}

tients with symptoms of HAE with normal C1-INH $(87.5 \%$ women) and 8 subjects asymptomatic for HAE ( $25 \%$ women). Mean age at onset of symptoms among the FXII-HAE patients was 13.8 years (range 6-25 years). Recurrent abdominal pain $(100 \%)$ and subcutaneous angioedema (87.5\%) were the most frequent clinical presentations. Two patients presented with associated laryngeal edema. In keeping with previous observations in patients with both C1-INH-HAE and HAE with normal C1-INH, all 7 women with FXII-HAE reported triggering or worsening of symptoms upon intake of estrogen-containing oral contraceptives and/or pregnancy. Conclusions: We report for the first time in Brazil a mutation in the $F 12$ gene as a likely cause of HAE with normal C1-INH in patients with recurrent attacks of angioedema and/or abdominal pain. A higher frequency of abdominal pain attacks and onset of symptoms at a younger age were observed among Brazilian patients when compared to those from other parts of the world.

ㄷ) 2015 S. Karger AG, Basel

\section{KARGER 125}

(c) 2015 S. Karger AG, Base

1018-2438/15/1662-0114\$39.50/0 


\section{Introduction}

Hereditary angioedema (HAE) is an autosomal dominant disorder characterized by edema of the skin, gastrointestinal tract and other organs. Clinically, it is characterized by episodes of swelling of the face, extremities and larynx, and recurrent attacks of severe abdominal pain [1-4]. The pathogenesis of classic HAE develops as a result of a genetic deficiency of the $\mathrm{C} 1$ inhibitor (C1-INH), a potent inhibitor of activation of the complement, coagulation and kinin pathways [5]. C1-INH deficiency results in activation of the kallikrein-kinin pathway with an increase in bradykinin production [6]. It has been documented that an increased release of bradykinin is the cause of angioedema via its action on B2 receptors leading to an increase in vascular permeability $[5,6]$.

More recently, a dominantly inherited disease has been described that has similar clinical characteristics to C1-INH-HAE, but is without abnormalities in antigenic and/or functional levels of C1-INH and has no mutation in the SERPING1 gene [7-9]. Two missense mutations, distributed across families with affected patients, were initially identified, located in exon 9 of the gene coding for coagulation factor XII (F12 gene). These mutations were designated as c.983C $>\mathrm{A}$ and c.983C $>\mathrm{G}$, corresponding to p.Thr328Lys and p.Thr328Arg, respectively, in association with HAE with normal C1-INH [10]. In the last few years, two additional mutations in the F12 gene also located in exon 9 have been reported in patients with HAE: a deletion of 72 base pairs (bp, i.e. c.971_1018+24 del72) [11] and a duplication of 18 bp (p.Pro298 Pro303dup) [12]. Initially known as HAE type III, HAE with normal C1-INH is currently classified into two subgroups: HAE with normal C1-INH and factor XII mutation (FXII-HAE) and HAE with normal C1-INH of unknown origin (U-HAE) [2]. To date, mutations in the F12 gene with a disease-causing role in $\mathrm{HAE}$ with normal C1INH have not been reported in Brazilian patients. Therefore, the aim of this study was to investigate the presence of F12 mutations in Brazilian patients with clinical characteristics of HAE with normal C1-INH.

\section{Subjects and Methods}

\section{Subjects}

Twenty-three individuals belonging to 5 unrelated families (Families 1-5) with index cases of female patients who presented with a history of recurrent angioedema with normal levels of C1$\mathrm{INH}$ and $\mathrm{C} 4$ were evaluated. Index patients from Families 4 and 5 also had normal functional activity of $\mathrm{C} 1-\mathrm{INH}$; however, this pa- rameter was not available for patients from Families 1-3. Patients were selected from those attending the Allergy Clinic of the Clinical Hospital of Ribeirão Preto Medical School or had been referred to our HAE Reference Center for clinical evaluation and/or genetic analysis. Information on an additional 31 subjects was obtained from close family members. Subjects aged between 18 and 71 years ( 15 females) were interviewed and underwent analysis for mutations in the F12 gene. Family pedigrees were constructed with 54 individuals distributed across up to four generations. Genetic evaluation was carried out in 10 members of Family 1, 4 members of Family 2, 3 members of Family 3, 4 members of Family 4 and 2 members of Family 5. The study was approved by the Ethics Committee of the Clinical Hospital of the Ribeirão Preto Medical School (Protocol No. 14521/2012), and all subjects or their caregivers gave written informed consent to participate in the study.

\section{HAE Severity Score}

A simplified HAE severity score was used, according to a previously described method [13]. Briefly, episodes of angioedema were classified according to the average frequency and intensity of symptoms before any treatment was started. The frequency of symptoms was quantitated as follows: 3 points for $>1$ episode a month, 2 points for between 6 and 11 episodes a year, 1 point for $<6$ episodes a year and 0 points for no symptoms of angioedema. The intensity of symptoms was defined as: the presence of discomfort but no disruption of daily activity ( 2 points), discomfort reducing normal daily activity (4 points) and an inability to work or perform daily activities and/or necessity of hospital care (5 points). The sum of the frequency and intensity scores was used to classify the severity of the disease as: severe ( $\geq 7$ points), moderate $(5 / 6$ points), mild ( $\leq 4$ points) and asymptomatic (0 points) [13].

\section{Genetic Analysis}

Genomic DNA was extracted from peripheral blood using the DNA Wizard genomic DNA purification kit (Promega, Madison, Wisc., USA), and the F12 gene was screened for mutations in exon 9. Sequencing of a PCR-amplified 363-bp DNA fragment comprising exon 9, intron 9 and part of exon 10 of the F12 gene was carried out in 23 individuals. PCR products were purified by the EXOSAPIT PCR product cleanup (Affymetrix, Cleveland, Ohio, USA) and sequenced using an ABI Prism 3500XL Genetic Analyzer (Applied Biosystems, Foster City, Calif., USA) and the BigDye ${ }^{\mathrm{TM}}$ Terminator V3.1 cycle sequencing kit (Applied Biosystems).

\section{Allelic Discrimination}

The c.983C >A (p.Thr328Lys) mutation was also screened by allelic discrimination. Sequence-specific primers and fluorogenic probes marked with FAM and VIC fluorophors were chosen with File Builder v3.1 software (Applied Biosystems). Genotyping for mutation c.983C $>$ A (p.Thr328Lys) was performed using a TaqMan-based allelic discrimination assay (Applied Biosystems), in $10-\mu \mathrm{l}$ reaction volumes. Allelic discrimination results were obtained from an Applied Biosystems 7700 sequence detection system.

\section{Levels of C1-INH and C4}

Serum levels of C1-INH and C4 were determined by nephelometry (Dade Behring, Newark, Del., USA), with normal values ranging from 0.21 to $0.39 \mathrm{~g} / \mathrm{l}$ and 0.1 to $0.4 \mathrm{~g} / \mathrm{l}$, respectively. Functional activity of C1-INH was assessed in outside clinical pathology laboratories, with normal values considered to be $80-130 \%$. 

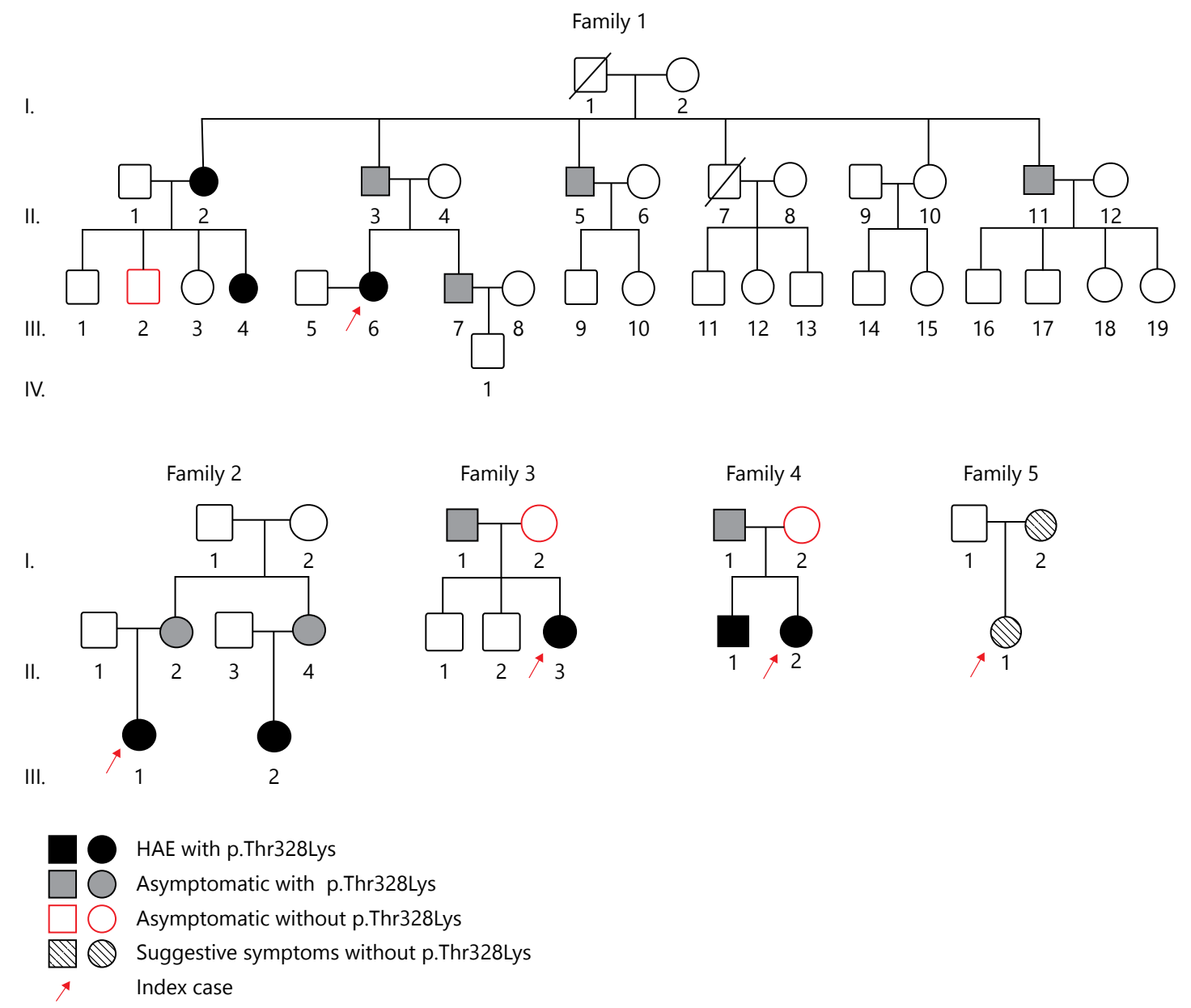

Fig. 1. Pedigrees of 5 Brazilian families with index patients who presented symptoms suggestive of HAE with normal C1-INH. Mutation p.Thr328Lys on exon 9 of the gene coding for coagula-

\section{Results}

\section{Identification of DNA Mutation}

The missense mutation c.983C $>$ A (p.Thr328Lys) of the F12 gene was identified in 16 subjects, belonging to 4 of the 5 families studied. Subjects with the p.Thr328Lys mutation included 8 patients with symptoms suggestive of HAE with normal C1-INH (7/8 women, $87.5 \%)$ and 8 subjects asymptomatic for $\mathrm{HAE}$ ( $2 / 8$ women, $25 \%$; fig. 1 ). The index patient in Family 5 (patient II.1) and her mother did not present the c.983C $>$ A mutation. The results of sequencing analysis were confirmed by allelic discrimination. tion factor XII (F12 gene) was identified in index cases and family members of $4 / 5$ Brazilian families with suspected hereditary angioedema with normal C1-INH, analyzed by DNA sequencing.

\section{Clinical Features}

Mean age at onset of symptoms among the 8 patients with FXII-HAE was 13.8 years (median 14 years), ranging from 6 to 25 years. Recurrent abdominal pain $(8 / 8$ patients, $100 \%)$ and subcutaneous angioedema (7/8 patients, $87.5 \%$ ) were the most frequent clinical presentations of the disease. Two patients (25\%) presented with associated laryngeal edema. All 7 women with FXIIHAE reported a triggering or worsening of symptoms upon intake of estrogen-containing oral contraceptives and/or pregnancy (table 1). As an example, we report the clinical features of patient II.2 from Family 4, an 18-yearold female with episodes of facial angioedema and severe 
Table 1. Clinical characteristics of patients and their relatives analyzed for mutations in exon 9 of the gene coding for coagulation factor XII

\begin{tabular}{|c|c|c|c|c|c|c|c|c|}
\hline & Sex & $\begin{array}{l}\text { Age, } \\
\text { years }\end{array}$ & $\begin{array}{l}\text { Age at onset } \\
\text { of symptoms, } \\
\text { years }\end{array}$ & Symptoms & $\begin{array}{l}\text { Clinical } \\
\text { score }^{\mathrm{b}}\end{array}$ & $\begin{array}{l}\text { Estrogen } \\
\text { sensitivity }\end{array}$ & $\begin{array}{l}\text { Prophylactic } \\
\text { treatment }\end{array}$ & $\begin{array}{l}\text { Mutation } \\
\text { c.983C }>\text { A } \\
\text { (p.Thr328Lys) }\end{array}$ \\
\hline II.2 & $\mathrm{F}$ & 51 & 25 & face, limb, abdominal attack & moderate & yes & tranexamic acid & yes \\
\hline II. 3 & M & 49 & n.a. & asymptomatic & asymptomatic & n.a. & n.a. & yes \\
\hline II. 5 & M & 45 & n.a. & asymptomatic & asymptomatic & n.a & n.a. & yes \\
\hline III.4 & $\mathrm{F}$ & 25 & 15 & face, limb, abdominal attack & moderate & yes & withdrawal of OC & yes \\
\hline III. $6^{\mathrm{a}}$ & $\mathrm{F}$ & 29 & 17 & $\begin{array}{l}\text { face, limb, abdominal attack, } \\
\text { upper respiratory tract }\end{array}$ & severe & yes & danazol & yes \\
\hline III.7 & M & 26 & n.a. & asymptomatic & asymptomatic & n.a. & n.a. & yes \\
\hline \multicolumn{9}{|c|}{ Family 2} \\
\hline \multicolumn{9}{|c|}{ Family 3} \\
\hline $\mathrm{I} .1$ & M & 59 & n.a. & asymptomatic & asymptomatic & n.a. & n.a. & yes \\
\hline I. 2 & $\mathrm{~F}$ & 61 & n.a. & asymptomatic & asymptomatic & n.a. & n.a. & no \\
\hline II. $3^{\mathrm{a}}$ & $\mathrm{F}$ & 27 & 7 & $\begin{array}{l}\text { face, limb, abdominal attack, } \\
\text { upper respiratory tract }\end{array}$ & severe & yes & tranexamic acid & yes \\
\hline \multicolumn{9}{|c|}{ Family 4} \\
\hline I. 1 & M & 46 & n.a. & asymptomatic & asymptomatic & n.a. & n.a. & yes \\
\hline I. 2 & $\mathrm{~F}$ & 46 & n.a. & asymptomatic & asymptomatic & n.a. & n.a. & no \\
\hline II.1 & M & 22 & 6 & $\begin{array}{l}\text { abdominal attack } \\
\text { (up to the age of } 15 \text { years) }\end{array}$ & moderate & n.a. & none & yes \\
\hline II. $2^{\mathrm{a}}$ & $\mathrm{F}$ & 18 & 16 & face, limb, abdominal attack & severe & yes & withdrawal of OC & yes \\
\hline
\end{tabular}

None of the patients was on hormone replacement therapy. 'Asymptomatic' describes patients without symptoms suggestive of HAE. OC = Oral contraceptives containing estrogen; n.a. = not applicable.

a Index case.

b According to Ferraro et al. [13].

${ }^{c}$ Yes: triggering and/or worsening symptoms upon estrogen exposure including use of OC or pregnancy, or remission of symptoms upon withdrawal of OC or the end of pregnancy. No: no effect of estrogen exposure on triggering and/or worsening of symptoms.

abdominal pain (table 1; fig. 1, 2). Symptoms had started when she was 16 years old, 15 days after initiation of use of estrogen-containing oral contraceptive. Ultrasonography and laparoscopy showed ascites during a severe episode of abdominal pain. A diagnosis was made 11 months after the onset of symptoms, and control of the symptoms was achieved by withdrawing the oral contraceptive. Her 22-year-old brother (patient II.1, table 1; fig. 1) reported having had episodes of abdominal pain during childhood. One of these episodes was severe, re- quiring admission to the hospital due to the suspicion of an acute abdominal condition. He has been asymptomatic since the age of 15 years. Patient II. 1 from Family 5 posed a clinical challenge, due to the lack of an F12 mutation. Despite extensive investigation and a variety of therapeutic strategies, it was not possible to define the etiopathogenesis of her severe symptoms of angioedema. The decision to treat her with danazol was based on the hypothesis she could have HAE with normal C1-INH, and several criteria were used: (1) a history of recurrent 


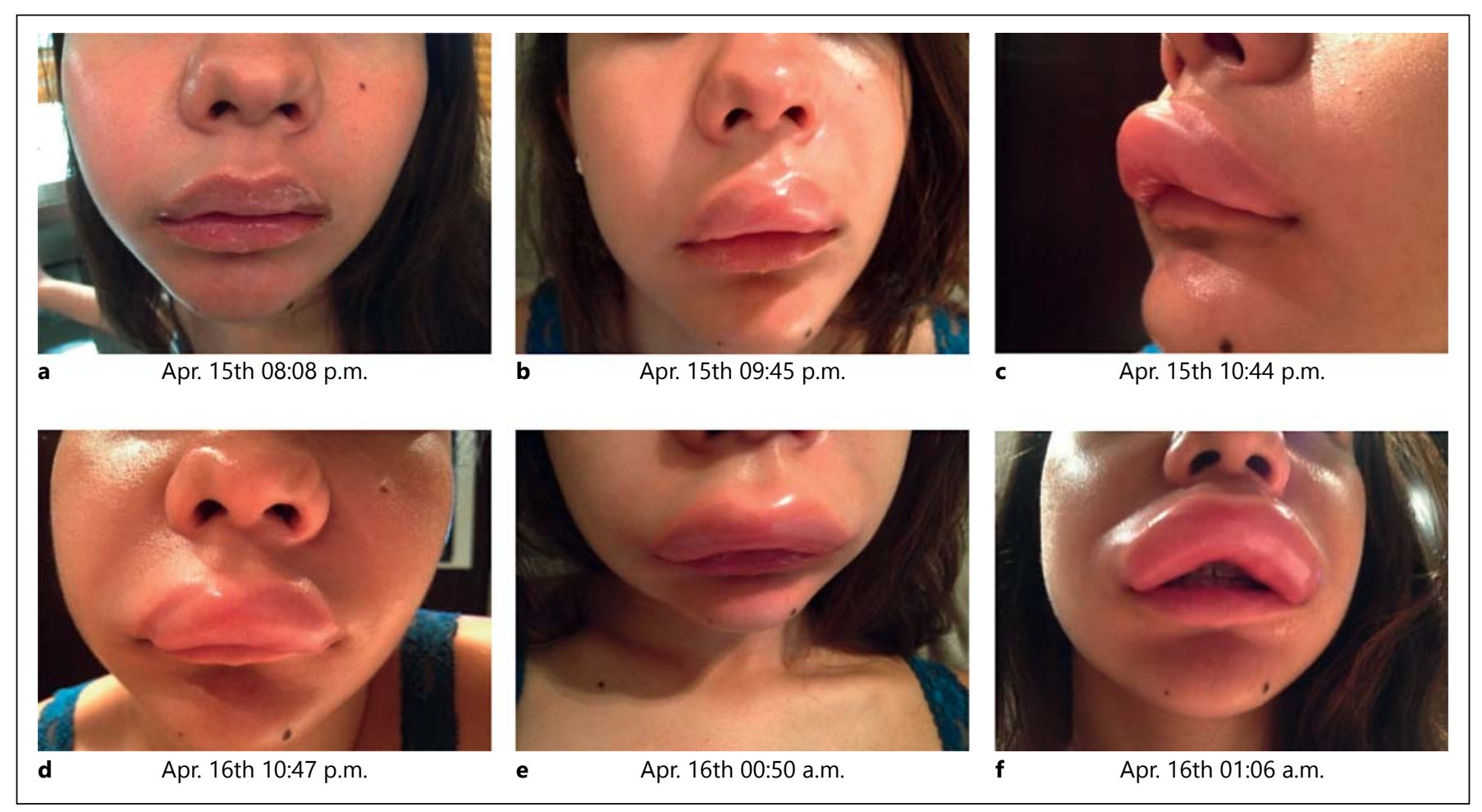

Fig. 2. Clinical presentation of HAE with normal C1-INH. a Onset of an attack of angioedema in an 18-year-old girl with HAE with normal C1-INH. b-f Time course of angioedema of lips and face.

angioedema episodes without urticaria, including laryngeal edema and abdominal pain attacks lasting 2-5 days, (2) her family history, i.e. a mother with recurrent face and limb edema without urticaria and no identifiable triggers, (3) a comprehensive work-up, with exclusion of other diagnoses, (4) normal levels and function of C1INH and normal C4 levels on several occasions and (5) no response to long-term, high-dose antihistamines and daily corticosteroids. These criteria have been proposed to help clinicians diagnose patients with HAE with normal C1-INH by the latest European Academy of Allergy and Clinical Immunology HAE consensus report [2]. With regard to our choice of treatment, there was no response to tranexamic acid in our patient. The use of danazol was a tentative investigation into the efficacy of a response to a more potent HAE prophylactic medication and at the beginning of treatment, she responded only to high doses (400-600 mg daily); now she has control of her symptoms with $200 \mathrm{mg} /$ day. Danazol has been shown to increase C1-INH messenger RNA expression in the peripheral blood mononuclear cells of HAE patients, even in the absence of a significant increase of C1INH plasma levels [14]; however, the C1-INH level and function were normal in our patient. Low plasma activity of aminopeptidase P (APP), a major metallopeptidase involved in kinin inactivation, particularly of the desArg9-bradykinin active metabolite, has been observed in the majority of patients with HAE, and APP activity showed an inverse correlation with disease severity [15]. Interestingly, danazol has also been shown to increase plasma APP activity in patients with HAE receiving prophylaxis with this attenuated androgen when compared to untreated patients [15]. It is possible that an increase in APP activity, contributing to more effective kinin inactivation, could be the mechanism by which danazol was beneficial to our patient.

\section{Discussion}

We report here the identification of the mutation c. $983 \mathrm{C}>\mathrm{A}$ (p.Thr328Lys) in exon 9 of the F12 gene, in patients presenting with clinical features of HAE with normal C1-INH belonging to 4 Brazilian families. Family members with the F12 gene mutation in the absence of symptoms suggestive of HAE could also be identified. 
These observations confirm the hereditary nature of this condition in our patients, and are in keeping with previous reports of a higher frequency of clinical disease among women, notably in association with increased estrogen exposure, as has already been described for C1-INHHAE $[9,16]$. In this context, genetic analysis was very helpful in facilitating a correct diagnosis and appropriate clinical management of most of our patients. In particular, our results reinforced the indication of medicines for the prophylaxis of HAE (e.g. danazol and tranexamic acid) and the recommendation of withdrawal of estrogen-containing oral contraceptives. In addition, confirmation of the diagnosis of FXII-HAE prompted us to prescribe HAE-specific medications for acute attacks, despite the knowledge that medications licensed for HAE are restricted to C1-INH-HAE, and that their use in other forms of angioedema is off-label [2]. Nonetheless, several case reports have shown success in treating acute attacks of angioedema in patients with HAE with normal C1-INH with icatibant, ecallantide and C1-INH concentrate $[2,9]$. Due to the life-threatening nature of the condition, we chose to indicate icatibant, the only HAE medication available in Brazil at the time of enrollment, as the medication for acute attacks.

The p.Thr328Lys had been previously identified in families with patients presenting with HAE with normal C1-INH from other parts of the world, particularly Europe [10, 17-23]. Patients who participated in our study and their relatives gave negative responses when asked about European or other foreign ancestry. However, we cannot rule out the possibility of a genetic input due to European or other ancestry, considering the fact that the population in Brazil is highly mixed [24, 25]. To our knowledge, there are no published studies reporting F12 gene mutations in Brazilian patients with HAE with normal C1-INH. This study underscores the fact that genetic analysis would be the only method to confirm the diagnosis of HAE with normal C1-INH, identifying patients with FXII-HAE. This method may, however, not be readily available to the practising allergist/immunologist in some areas of the world.

It is assumed that C1-INH-HAE and HAE with normal C1-INH have similar clinical behavior, with bradykinin as the key mediator of angioedema in these two conditions $[9,26]$. Affected women often get worse when on estrogen-containing medication or during pregnancy [26], and symptoms show no improvement with antihistamines and corticosteroids in both C1-INH-HAE and HAE patients with normal C1-INH [9]. However, a few clinical differences have been reported between patients

F12 Mutation in Brazilian Families with HAE with Normal C1-INH with HAE with normal C1-INH and those with C1-INHHAE, including: fewer attacks with more attack-free intervals, more frequent occurrence of cutaneous, facial, tongue and uvula edema and of asphyxiation by laryngeal attacks, a lower percentage of recurrent abdominal pain attacks (in approx. $50 \%$ of patients) $[9,26]$, no prodromical erythema marginatum and onset of symptoms at an older age (mean 27 years) $[9,16]$. Patients with HAE who participated in our study presented most of these characteristics, with the exception of a higher frequency of abdominal pain attacks (observed in $100 \%$ of the patients) sometimes associated with the presence of ascites, and an earlier onset of symptoms (mean 13.8 years). A relevant observation was that one child presented with recurrent abdominal pain as the only symptom of FXIIHAE, and some patients' symptoms started with abdominal attacks in childhood, as has been described for C1INH-HAE patients $[13,27]$. Therefore, our data provide support for a recommendation that both C1-INH-HAE and HAE with normal C1-INH should be included in the differential diagnosis of recurrent abdominal pain, particularly in patients with a positive family history of angioedema.

In conclusion, we have described the identification of the FXII mutation p.Thr328Lys in Brazilian patients with HAE with normal C1-INH. Although our study included only a small number of patients, the results suggest that clinical aspects among Brazilian patients may differ from those reported in other areas of the world, including a higher frequency of abdominal pain attacks and onset of symptoms at a younger age. Patients with U-HAE remain a challenge in clinical practice. However, their clinical features resemble those of C1-INH HAE and FXII-HAE patients, including recurrent episodes of subcutaneous edema without urticaria, abdominal pain and laryngeal edema, poor or no response to treatment with antihistamines and corticosteroids, a family history suggestive of HAE and the triggering and/or worsening of symptoms with exposure to estrogen. Provided other conditions have been excluded, such clinical features should prompt the assisting physician to consider treatment with HAEspecific agents both for acute attacks and prophylaxis. In fact, the recent consensus document on HAE [2] cites C1INH agents, icatibant, ecallantide, progesterone, danazol and tranexamic acid as potential therapeutic options for U-HAE and FXII-HAE patients. It would be desirable to have controlled studies to support clinical decisions. Certainly, future studies will be necessary to identify the molecular pathways involved in causing symptoms in patients with U-HAE. 


\section{Acknowledgments}

We are very grateful to Prof. Thomas A.E. Platts-Mills, Asthma and Allergic Diseases Center, University of Virginia, Charlottesville, Va., USA, for critical reading of the manuscript. We wish to thank Mrs. Luana S.M. Maia, Mrs. Rosangela Prendim Tortora and Mrs. Adriana Aparecida Marques for technical assistance, and the patient with HAE for sharing her pictures of an angioedema attack and for allowing us to reproduce them in this paper. Finan- cial support was received from the Foundation for Research Support of the State of São Paulo (FAPESP) and Institute for Investigation in Immunology iii-INCT, Brazil. A.S.M. is supported by a postdoctoral scholarship by FAPESP.

\section{Disclosure Statement}

The authors have no conflict of interest to declare.

\section{References}

1 Bork K: Angioedema. Immunol Allergy Clin North Am 2014;34:23-31.

2 Cicardi M, Aberer W, Banerji A, Bas M, Bernstein JA, Bork K, Caballero T, Farkas $\mathrm{H}$, Grumach A, Kaplan AP, Riedl MA, Triggiani M, Zanichelli A, Zuraw B, on behalf of HAWK under the patronage of EAACI: Classification, diagnosis, and approach to treatment for angioedema: consensus report from the $\mathrm{He}$ reditary Angioedema International Working Group. Allergy 2014;69:602-616.

3 Craig T, Aygoren-Pursun E, Bork K, Bowen T, Boysen H, Farkas H, Grumach A, Katelaris $\mathrm{CH}$, Lockey R, Longhurst H, Lumry W, Magerl M, Martinez-Saguer I, Ritchie B, Nast A, Pawankar R, Zuraw B, Maurer M: WAO guideline for the management of hereditary angioedema. World Allergy Organ J 2012;5: 182-199.

4 Longhurst H, Cicardi M: Hereditary angiooedema. Lancet 2012;379:474-481.

5 Kaplan AP, Joseph K: Pathogenic mechanisms of bradykinin mediated diseases: dysregulation of an innate inflammatory pathway. Adv Immunol 2014;121:41-89.

6 Maurer M, Bader M, Bas M, Bossi F, Cicardi M, Cugno M, Howarth P, Kaplan A, Kojda G, Leeb-Lundberg F, Lötvall J, Magerl M: New topics in bradykinin research. Allergy 2011; 66:1397-1406.

7 Bork K, Barnstedt SE, Koch P, Traupe H: Hereditary angioedema with normal $\mathrm{C} 1$ inhibitor activity in women. Lancet 2000;356:213-217.

8 Zuraw BL, Bork K, Binkley KE, Banerji A, Christiansen SC, Castaldo A, Kaplan A, Riedl M, Kirkpatrick C, Magerl M, Drouet C, Cicardi M: Hereditary angioedema with normal C1 inhibitor function: consensus of an international expert panel. Allergy Asthma Proc 2012;33(suppl 1):S145-S156.

9 Riedl MA: Hereditary angioedema with normal C1-INH (HAE type III). J Allergy Clin Immunol Pract 2013;1:427-432.

10 Dewald G, Bork K: Missense mutations in the coagulation factor XII (Hageman factor) gene in hereditary angioedema with normal $\mathrm{C} 1$ inhibitor. Biochem Biophys Res Commun 2006; 343:1286-1289.
11 Bork K, Wulff K, Meinke P, Wagner N, Hardt J, Witzke G: A novel mutation in the coagulation factor 12 gene in subjects with hereditary angioedema and normal $\mathrm{C} 1$ inhibitor. Clin Immunol 2011;141:31-35.

12 Kiss N, Barabás E, Várnai K, Halász A, Varga LÁ, Prohászka Z, Farkas H, Szilágyi Á: Novel duplication in the $F 12$ gene in a patient with recurrent angioedema. Clin Immunol 2013; 149:142-145.

13 Ferraro MF, Moreno AS, Castelli EC, Donadi EA, Palma MS, Arcuri HA, Lange AP, Bork K, Sarti W, Arruda LK: A single nucleotide deletion at the $\mathrm{C} 1$ inhibitor gene as the cause of hereditary angioedema: insights from a Brazilian family. Allergy 2011;66:1384-1390.

14 Pappalardo E, Zingale LC, Cicardi M: Increased expression of C1-inhibitor mRNA in patients with hereditary angioedema treated with danazol. Immunol Lett 2003;86:271-276.

15 Drouet C, Desormeaux A, Robillard J, Ponard D, Bouillet L, Martin L, Kanny G, MoneretVautrin DA, Bosson JL, Quesada JL, LopezTrascasa M, Adam A: Metallopeptidase activities in hereditary angioedema: effect of androgen prophylaxis on plasma aminopeptidase $\mathrm{P}$. J Allergy Clin Immunol 2008;121:429-433.

16 Bork K: Hereditary angioedema with normal C1 inhibitor. Immunol Allergy Clin North Am 2013;33:457-470.

17 Martin L, Raison-Peyron N, Nothen MM, Cichon S, Drouet C: Hereditary angioedema with normal $\mathrm{C} 1$ inhibitor gene in a family with affected women and men is associated with the p.Thr328Lys mutation in the F12 gene. J Allergy Clin Immunol 2007;120:975977.

18 Bork K: Hereditary angioedema with normal $\mathrm{C} 1$ inhibitor activity including hereditary angioedema with coagulation factor XII gene mutations. Immunol Allergy Clin North Am 2006;26:709-724.

19 Gomez-Traseira C, Lopez-Lera A, Drouet C, Lopez-Trascasa M, Perez-Fernandez E, Favier B, Prior N, Caballero T: Hereditary angioedema caused by the p.Thr309Lys mutation in the F12 gene: a multifactorial disease. J Allergy Clin Immunol 2013;132:986-989.
20 Vitrat-Hincky V, Gompel A, Dumestre-Perard C, Boccon-Gibod I, Drouet C, Cesbron JY, Lunardi J, Massot C, Bouillet L: Type III hereditary angio-oedema: clinical and biological features in a French cohort. Allergy 2010; 65:1331-1336.

21 Charignon D, Ghannam A, Defendi F, Ponard D, Monnier N, López Trascasa M, Launay D, Caballero T, Djenouhat $\mathrm{K}$, Fain $\mathrm{O}$, Cichon S, Martin L, Drouet C: Hereditary angioedema with F12 mutation: factors modifying the clinical phenotype. Allergy 2014;69: 1659-1665.

22 Marcos C, Lopez Lera A, Varela S, Linares T, Alvarez-Eire MG, Lopez-Trascasa M: Clinical, biochemical, and genetic characterization of type III hereditary angioedema in 13 northwest Spanish families. Ann Allergy Asthma Immunol 2012;109:195200.e192.

23 Bork K, Wulff K, Hardt J, Witzke G, Staubach $\mathrm{P}$ : Hereditary angioedema caused by missense mutations in the factor XII gene: clinical features, trigger factors, and therapy. J Allergy Clin Immunol 2009;124:129-134.

24 Pena SD, Di Pietro G, Fuchshuber-Moraes M, Genro JP, Hutz MH, Kehdy FeS, Kohlrausch F, Magno LA, Montenegro RC, Moraes MO, de Moraes ME, de Moraes MR, Ojopi EB, Perini JA, Racciopi C, RibeiroDos-Santos AK, Rios-Santos F, Romano-Silva MA, Sortica VA, Suarez-Kurtz G: The genomic ancestry of individuals from different geographical regions of Brazil is more uniform than expected. PLoS One 2011;6: e17063.

25 Pena SD, Bastos-Rodrigues L, Pimenta JR, Bydlowski SP: DNA tests probe the genomic ancestry of Brazilians. Braz J Med Biol Res 2009; 42:870-876.

26 Bork K: Diagnosis and treatment of hereditary angioedema with normal $\mathrm{C} 1$ inhibitor. Allergy Asthma Clin Immunol 2010;6:15.

27 Bork K, Staubach P, Eckardt AJ, Hardt J: Symptoms, course, and complications of abdominal attacks in hereditary angioedema due to $\mathrm{C} 1$ inhibitor deficiency. Am J Gastroenterol 2006;101:619-627. 